\title{
Phase Shift between Supersymmetric Partner Potentials
}

\author{
Sabyasachi Mahapatra \\ Department of Physics, St. Paul's Cathedral Mission College, Raja Rammohan Roy Sarani, Kolkata, India \\ Email: smahapatra99@gmail.com
}

Received July 23, 2011; revised September 6, 2011; accepted October 15, 2011

\begin{abstract}
The relationship between phase shifts produced by two supersymmetric (SUSY) partner potentials has been examined critically. Phase shifts produced by two SUSY partner potentials bear a simple relation irrespective of being shape invariant potential (SIP) or not (non-SIP). In general, the phase shift cannot be obtained algebraically, even for standard SIPs except the Coulomb potential.
\end{abstract}

Keywords: Phase Shift; Supersymmetry; Shape Invariant Potential

\section{Introduction}

Supersymmetric quantum mechanics (SSQM) was introduced by Witten [1], Coopper and Freedman [2] as a simple model for testing supersymmetric field theories which were introduced by Gel'fand and Likhtman [3] for a unified description of the fundamental interaction in nature. The study of SSQM has since been of great interest, as it provided an insight into the factorization of the Hamiltonian and introduction of partner Hamiltonians having wholly or partially identical energy spectrum. It also explained analytical solvability of potentials, whose partner potentials have the same shape, introducing the concept of shape invarince. From a given Hamiltonian $\left(H_{1}\right)$, a partner Hamiltonian $\left(H_{2}\right)$ is usually obtained by deleting the ground state of $H_{1}$, the rest of the spectrum of $H_{1}$ being identical with the spectrum of $H_{2}$ [4]. The partner Hamiltonian $\mathrm{H}_{2}$ can also be obtained by adding an energy level below the ground state of $H_{1}$ or by matching the spectrum of $H_{2}$ identical to that of $H_{1}$ (isospectral Hamiltonians) [5]. We will focus our attention to the first class of partner Hamiltonians. An elegant and exhaustive review of the procedure and its consequences can be found in Ref. [4]. The principal result is the energy degeneracy, viz., $E_{n+1}^{(1)}=E_{n}^{(2)},(n=0,1,2, \cdots)$, where $E_{n}^{(i)}$ is the energy of the $n$-th excited (bound) state of $H^{(i)}$. The bound state eigen function $\psi_{n}^{(2)}$ of $H^{(2)}$ can be obtained by applying an operator $A$ on the eigen function $\psi_{n+1}^{(1)}$ of $H^{(1)}$, viz., $\psi_{n}^{(2)} \propto A \psi_{n+1}^{(1)}$. The operator $A$ destroys a node of an eigen function of $H^{(1)}$. This procedure can also be extended to the scattering (unbound) states. From the asymptotic form of the scattering wave function, one can then find a relation between the phase shifts produced by the two partner potentials [5]. If the potential $V^{(2)}$ of the partner Hamiltonian
$H^{(2)}$ has the same functional shape (but with different parameters) as that of the potential $V^{(1)}$ of the original Hamiltonian $H^{(1)}$, then the potential is said to be a "shape invariant potential (SIP)". It can be shown that the entire bound state energy spectrum and corresponding energy eigen functions of $H^{(1)}$ can be obtained algebraically if $V^{(1)}$ is shape invariant [4]. However, shape invariance is not a prerequisite for obtaining the partner potential $V^{(2)}$ from any starting potential $V^{(1)}$. The partner potential can be constructed, if the ground state wave function of the original potential is known, even if the corresponding Hamiltonian is not solvable analytically. Hence for any given potential $V^{(1)}$, one can construct the partner potential $V^{(2)}$, according to a clear prescription (to be outlined in Section 2), such that $V^{(2)}$ has the same energy spectrum as $V^{(1)}$, except that the ground state of $V^{(1)}$ will be absent in the spectrum of $V^{(2)}$. Then extending the procedure to scattering states, one can show that the phase shifts produced by $V^{(1)}$ and $V^{(2)}$ bear a simple relation [5], which will also be outlined in Section 2. Since this procedure is valid for any potential $V^{(1)}$, the relationship between the phase shifts produced by $V^{(1)}$ and $V^{(2)}$ should also hold whether or not $V^{(1)}$ is shape invariant. Likewise, energy degeneracy $\left(E_{n+1}^{(1)}=E_{n}^{(2)}\right)$ and relation between $\psi_{n+1}^{(1)}$ and $\psi_{n}^{(2)}$ should also hold even if $V^{(1)}$ is not shape invariant. In Reference [5], it was shown analytically that the phase shift relation holds for the Coulomb potential, which is well known to be shape invariant. In connection with that derivation, to be outlined in Section 2.2, it was shown that if the original potential $V^{(1)}$ is taken with an orbital angular momentum $l$, then the phase shift of the partner potential corresponds to angular momentum $(l+1)$. For the Coulomb potential (an SIP), the partner 
of $\left(-\frac{e^{2}}{r}+\frac{\hbar^{2}}{2 m} \frac{l(l+1)}{r^{2}}\right)$ is

$$
\left[-\frac{e^{2}}{r}+\frac{\hbar^{2}}{2 m} \frac{(l+1)(l+2)}{r^{2}}+\left(\varepsilon_{0 l}-\varepsilon_{1 l}\right)\right],
$$

where $\varepsilon_{n l}$ is the energy of the eigen state with radial and orbital quantum numbers $n$ and $l$ respectively. In this case the partner potential has an explicit orbital angular momentum $(l+1)$. It is important to note that the orbital angular momentum $(l)$ becomes the shape invariance parameter, and it changes to $(l+1)$ by the condition of shape invariance

$$
V^{(2)}\left(r ; a_{1}=l\right)=V^{(1)}\left(r ; a_{2}=l+1\right)+R\left(a_{1}\right)
$$

with $\quad a_{2}=f\left(a_{1}\right)=l+1$.

Consequently, the phase shifts associated with $V^{(2)}$ corresponds to orbital angular momentum $(l+1)$. On the other hand, for a non-SIP, there is no shape invariance condition, Equation (1). Hence $V^{(2)}$ is not explicitly associated with orbital angular momentum $(l+1)$. It is actually associated with orbital angular moentum $l$, but its $r \rightarrow 0$ behavior is $(l+1)(l+2) / r^{2} \quad$ (as will be shown in Section 2.2). This may cause a serious pitfall in assigning orbital angular momentum for the partner potential $V^{(2)}$ : if an explicit $(l+1)$ is taken for $V^{(2)}$, it will give wrong results. Instead, one should include $l$ in $V^{(1)}$ only and then calculate its partner $V^{(2)}$, without additional inclusion of any $l$. We wish to stress this point in this paper, to avoid a possible pitfall.

In the present work, we critically examine the relationship between phase shifts produced by the two partner potentials for both SIP and non-SIP. We find that the difference between the phase shifts produced by $V^{(1)}$ and $V^{(2)}$ for both SIP and non-SIP do not obey the SSQM predicted relation, if $V^{(1)}$ and its supersymmetric partner $V^{(2)}$ are taken with $l$ and $(l+1)$ respectively, whereas it is obeyed when both $V^{(1)}$ and $V^{(2)}$ are taken with the same $l$. Thus, unless the parameter involved in the shape invariance relation, Equation (1), change automatically from $l$ to $(l+1)$, one should not change $l$ for the partner.

The paper is organized as follows. In Section 2, we briefly recapitulate the basic relations between energies, eigen functions and phase shifts of the partner potentials. We also indicate how the partner potential can be constructed for any potential, if its ground state wave function is known. In Section 3, results will be presented for the finite square well potential, which is a non-SIP, along with the Pöschl-Teller potential (SIP). The difference of their phase shifts will be compared with the expectations from SSQM. In Section 4, we draw our conclusions.

\section{Partner Potentials: Relations between Their Observables}

\subsection{Energy Degeneracy and Shape Invariance}

In this sub-section, we present an outline of energy degeneracy associated with partner potentials, following the review article of Cooper, Khare and Sukhatme [4]. Consider a spherically symmetric potential $V^{(1)}(r)$. The energy scale is so chosen that the ground state of orbital angular momentum $l$ in this potential has zero energy. We assume that $V^{(1)}(r)$ goes slower than $r^{-2}$ for $r \rightarrow 0$ and approaches a constant value $V_{\infty}$ for $r \rightarrow \infty$. Then the Schrödinger equation for the ground state of orbital angular momentum $l$ in this potential is

$$
H^{(1)} \psi_{0}^{(1)}(r)=\left[-\frac{\hbar^{2}}{2 m} \frac{\mathrm{d}^{2}}{\mathrm{~d} r^{2}}+V_{\text {eff }}^{(1)}(r)\right] \psi_{0}^{(1)}(r)=0 .
$$

where, $V_{\text {eff }}^{(1)}(r)=\frac{\hbar^{2}}{2 m} \frac{l(l+1)}{r^{2}}+V^{(1)}(r)$. Then one can obtain $V_{\text {eff }}^{(1)}(r)$ as

$$
V_{e f f}^{(1)}(r)=\frac{\hbar^{2}}{2 m} \frac{\psi_{0}^{(1) "}(r)}{\psi_{0}^{(1)}(r)} .
$$

Define a "super potential" $W(r)$ through

$$
W(r)=-\frac{\hbar}{\sqrt{2 m}} \frac{\psi_{0}^{(1)^{\prime}}(r)}{\psi_{0}^{(1)}(r)},
$$

so that

$$
V_{e f f}^{(1)}(r)=W^{2}(r)-\frac{\hbar}{\sqrt{2 m}} W^{\prime}(r) .
$$

Define a partner potential

$$
V_{\text {eff }}^{(2)}(r)=W^{2}(r)+\frac{\hbar}{\sqrt{2 m}} W^{\prime}(r),
$$

and the corresponding Hamiltonian

$$
H^{(2)}=\left[-\frac{\hbar^{2}}{2 m} \frac{\mathrm{d}^{2}}{\mathrm{~d} r^{2}}+V_{e f f}^{(2)}(r)\right] .
$$

The supersymmetric partner of $V_{\text {eff }}^{(1)}(r)$ is $V_{\text {eff }}^{(2)}(r)$. Defining the operators $A$ and $A^{\dagger}$ as

$$
A=\frac{\hbar}{\sqrt{2 m}} \frac{\mathrm{d}}{\mathrm{d} r}+W(r), \quad A^{\dagger}=-\frac{\hbar}{\sqrt{2 m}} \frac{\mathrm{d}}{\mathrm{d} r}+W(r),
$$

one sees that

$$
H^{(1)}=A^{\dagger} A, \quad H^{(2)}=A A^{\dagger} .
$$

The eigenvalue equations satisfied by these Hamiltonians are

$$
\begin{aligned}
& H^{(1)} \psi_{n}^{(1)}(r)=A^{\dagger} A \psi_{n}^{(1)}(r)=E_{n}^{(1)} \psi_{n}^{(1)}(r), \\
& H^{(2)} \psi_{n}^{(2)}(r)=A A^{\dagger} \psi_{n}^{(2)}(r)=E_{n}^{(2)} \psi_{n}^{(2)}(r) .
\end{aligned}
$$


Then one can easily see that (energies are expressed in the scale in which $\left.E_{0}^{(1)}=0\right)$ [4]

$$
\begin{aligned}
& E_{n}^{(2)}=E_{n+1}^{(1)}, \quad \psi_{n}^{(2)}=\left[E_{n+1}^{(1)}\right]^{-1 / 2} A \psi_{n+1}^{(1)}, \\
& \psi_{n+1}^{(1)}=\left[E_{n}^{(2)}\right]^{-1 / 2} A^{\dagger} \psi_{n}^{(2)} .
\end{aligned}
$$

This shows that $H^{(2)}$ has the same spectrum as that of $H^{(1)}$, except that there is no state in $H^{(2)}$ corresponding to the ground state of $H^{(1)}$.

If $V_{\text {eff }}^{(2)}(r)$ has the same functional shape as that of $V_{\text {eff }}^{(1)}(r)$, but have different parameters, then the potential is said to be a shape invariant potential (SIP). The parameters of the partner are obtained as a simple mathematical function of the original parameters of $V_{\text {eff }}^{(1)}(r)$. Then it is easily seen that the entire energy spectrum is obtained algebraically [4].

From Equations (4)-(6), we have

$$
\begin{aligned}
V_{e f f}^{(2)}(r)= & \frac{\hbar^{2}}{m} \frac{(l+1)(l+2)}{2 r^{2}}+V^{(1)}(r) \\
& -\frac{\hbar^{2}}{m} \frac{\mathrm{d}^{2}}{\mathrm{~d} r^{2}}\left[\ln \left\{\frac{\psi_{0}^{(1)}(r)}{r^{l+1}}\right\}\right] .
\end{aligned}
$$

This shows that the $r \rightarrow 0$ behavior of $V_{\text {eff }}^{(2)}(r)$ corresponds to angular momentum $l+1$, since for $r \rightarrow 0$, $\psi_{0}^{(1)}(r)$ goes as $r^{l+1}$. Both the potentials $V_{\text {eff }}^{(1)}(r)$ and $V_{\text {eff }}^{(2)}(r)$ approach the same value $V_{\infty}$ in the limit $r \rightarrow \infty$.

From Equations (4) and (8), we have

$$
A=\frac{\hbar}{\sqrt{2 m}}\left[\frac{\mathrm{d}}{\mathrm{d} r}-\frac{\mathrm{d}}{\mathrm{d} r}\left\{\ln \left(\psi_{0}^{(1)}(r)\right)\right\}\right] .
$$

The operator $A$ takes an eigenfunction of $H^{(1)}$ into an eigenfunction of $H^{(2)}$ (including those corresponding to continuous eigenvalues).

\subsection{Relation between the Phase Shifts Produced by the Partner Potentials}

For scattering states, energy should be in the continuum. In the energy scale chosen so far, $E_{0}^{(1)}=0$ and $\lim _{r \rightarrow \infty} V^{(1)}(r)=V_{\infty}$. Now we change our energy scale by shifting it by $-V_{\infty}$, such that each one of $V^{(1)}(r)$, $V^{(2)}(r), V_{\text {eff }}^{(1)}(r)$ and $V_{\text {eff }}^{(2)}(r)$ vanish in the limit $r \rightarrow \infty$. Energies of the bound states are expressed as

$$
\varepsilon_{n}^{(i)}=E_{n}^{(i)}-V_{\infty}(i=1,2 ; n=0,1,2, \cdots)
$$

We consider scattering states $\psi_{E}^{(1)}(r)$ and $\psi_{E}^{(2)}(r)$ corresponding to energy $E=\left(\hbar^{2} k^{2}\right) / 2 m \quad(E>0)$ in the potentials $V_{\text {eff }}^{(1)}(r)$ and $V_{\text {eff }}^{(2)}(r)$ respectively. These states are connected by the operator $A$

$$
\begin{aligned}
& \psi_{E}^{(2)}(r) \propto A \psi_{E}^{(1)}(r) \\
= & \frac{\hbar}{\sqrt{2 m}}\left[\frac{\mathrm{d}}{\mathrm{d} r}-\frac{\mathrm{d}}{\mathrm{d} r}\left\{\ln \left(\psi_{0}^{(1)}(r)\right)\right\}\right] \psi_{E}^{(1)}(r) .
\end{aligned}
$$

To calculate phase shifts, we need the asymptotic forms of these wave functions. For $r \rightarrow \infty$,

$$
\psi_{0}^{(1)} \propto \exp \left(-\gamma_{0} r\right),
$$

where

$$
\gamma_{0}=\sqrt{2 \frac{m}{\hbar^{2}}\left(-\varepsilon_{0}^{(1)}\right)} .
$$

Hence we have

$$
\psi_{E}^{(2)}(r) \propto \frac{\hbar}{\sqrt{2 m}}\left[\frac{\mathrm{d}}{\mathrm{d} r}+\gamma_{0}\right] \psi_{E}^{(1)}(r) .
$$

The asymptotic form of $\psi_{E}^{(1)}(r)$ is

$$
\psi_{E}^{(1)}(r) \propto \sin \left[k r-\frac{l \pi}{2}+\delta_{l}^{(1)}(k)\right],
$$

where $\delta_{l}^{(1)}(k)$ is the phase shift produced by $V_{\text {eff }}^{(1)}(r)$, corresponding to orbital angular momentum $l$ and energy $E=\frac{\hbar^{2} k^{2}}{2 m}$. Substituting it in Equation (17), we have

$$
\psi_{E}^{(2)}(r) \propto \sin \left[k r-\frac{(l+1) \pi}{2}+\delta_{l+1}^{(2)}(k)\right],
$$

where

$$
\delta_{l+1}^{(2)}(k)=\delta_{l}^{(1)}(k)-\tan ^{-1}\left(\frac{\gamma_{0}}{k}\right)
$$

is the phase shift produced by $V_{\text {eff }}^{(2)}(r)$, corresponding to energy $E=\frac{\hbar^{2} k^{2}}{2 m}$. Note that the $r \rightarrow 0$ behavior of the partner potential corresponds to orbital angular momentum $l+1$ [see the discussion following Equation (12)], which justifies the subscript $(l+1)$ of $\delta^{(2)}$. Note also that $\delta_{l+1}^{(2)}(k)$ is the phase shift produced by $V_{\text {eff }}^{(2)}(r)$, which is the supersymmetric partner of $V_{\text {eff }}^{(1)}(r)$, and not of the potential $\left[V^{(2)}(r)+\frac{\hbar^{2}}{2 m} \frac{(l+1)(l+2)}{r^{2}}\right]$ (where $V^{(2)}(r)$ is the supersymmetric partner of $\left.V^{(1)}(r)\right)$, even though $\delta_{l+1}^{(2)}(k)$ corresponds to the phase shift of the $(l+1)$-th partial wave. Thus it will be wrong to take the phase shift of the explicit $(l+1)$-th partial wave of $V^{(2)}(r)$, unless $l$ is the shape invariance parameter of Equation (1), such that $f(l)=l+1$. For a shape invariant potential, for which Equation (1) holds, $\delta_{l}^{(1)}(k)$ and $\delta_{l+1}^{(2)}(k)$ correspond to the same functional form of the potential. Hence in this case, use of Equation (20) permits the derivation of an algebraic expression for the phase shift. This is true only for the Coulomb potential $[5,7]$. In the above derivation, we have not assumed the 
shape invariance property [4]. Hence, Equation (20) should hold equally well for both SIP and non-SIP.

\section{Results}

\subsection{Application to a Square Well Potential}

As a simple application, we consider the $l=0$ partial wave in a square well potential having the form

$$
V(r) \begin{cases}=-V_{0}, & r \leq R \\ =0, & r>R .\end{cases}
$$

Note that the finite square well potential, although solvable semi-analytically, is not an SIP. A bound state in this potential is obtained using the continuity of the wave function and its first derivative at $r=R$. This gives a transcendental equation for the binding energy $B\left(=-E_{B}, E_{B}<0\right)$ :

$$
\beta \tan K R+K=0
$$

where

$$
K=\sqrt{\frac{2 m\left(V_{0}-B\right)}{\hbar^{2}}}, \quad \beta=\sqrt{\frac{2 m B}{\hbar^{2}}} .
$$

The numerical solution of Equation (21) with the largest possible value of $B\left(=B_{0}\right.$, say $)$ gives binding energy of the ground state. Corresponding to this value of $B_{0}$, we can get the ground state wave function $\psi_{0}^{(1)}(r)$. Then the superpotential for the square well is given by Equation (3) as

$$
W(r) \begin{cases}=-\frac{\hbar K_{0}}{\sqrt{2 m}} \cot K_{0} r, \quad(r \leq R) \\ =\frac{\hbar \beta_{0}}{\sqrt{2 m}} \quad(r>R)\end{cases}
$$

where $K_{0}$ and $\beta_{0}$ are values of $K$ and $\beta$ respectively, with $B$ replaced by $B_{0}$. Substitution of $W(r)$ in Equation (5) reproduces $V_{\text {eff }}^{(1)}(r)$ (for $l=0$ ) and Equation (6) gives

$$
V_{\text {eff }}^{(2)}(r) \begin{cases}=\frac{\hbar^{2}}{2 m} K_{0}^{2} \cot ^{2} K_{0} r+\frac{\hbar^{2}}{2 m} K_{0}^{2} \operatorname{cosec}^{2} K_{0} r, & r \leq R \\ =\frac{\hbar^{2}}{2 m} \beta_{0}^{2} . & r>R\end{cases}
$$

\subsection{Numerical Calculation of the Phase Shift}

For the potential $V^{(1)}(r)$ (with $V_{\infty}$ subtracted, so that $V^{(1)}(r)$ vanishes asymptotically) the radial Schrödinger equation for the $l$-th partial wave takes the form

$$
\left[-\frac{\hbar^{2}}{2 m} \frac{\mathrm{d}^{2}}{\mathrm{~d} r^{2}}+V_{\text {eff }}^{(1)}(r)-E\right] \psi_{E}^{(1)}(r)=0 .
$$

The asymptotic solution is given by Equation (18). We integrate Equation (24) numerically using the RungaKutta algorithm from $r \rightarrow 0$ (subject to $\psi_{E}^{(1)}(0)=0$ ) to two large values of $r$ (say $R_{1}$ and $R_{2}$ ) to obtain $\phi_{1}=\psi_{E}^{(1)}\left(R_{1}\right)$ and $\phi_{2}=\psi_{E}^{(1)}\left(R_{2}\right)$. Then

$$
\delta_{l}^{(1)}(k)=\tan ^{-1}\left[\frac{\phi_{2} \sin \left(k R_{1}-\frac{l \pi}{2}\right)-\phi_{1} \sin \left(k R_{2}-\frac{l \pi}{2}\right)}{\phi_{1} \cos \left(k R_{2}-\frac{l \pi}{2}\right)-\phi_{2} \cos \left(k R_{1}-\frac{l \pi}{2}\right)}\right]
$$

For calculation of $\delta_{l+1}^{(2)}(k)$, we follow the above procedure, replacing $V_{\text {eff }}^{(1)}(r)$ by $V_{\text {eff }}^{(2)}(r)$ in Equaiton (24). The partner potential is calculated using Equations (2), (4) and (6). No explicit l-dependent term is inserted in $V_{\text {eff }}^{(2)}(r)$.

\subsection{Numerical Results}

We choose dimensionless units: $\hbar=\sqrt{2 m}=1$ and take $V_{0}=50, R=1$, which support two bound states for $l=0$ in this square well potential. Numerical solution of Equation (21) gives the binding energies of the ground and first excited state as $B_{0}=42.374903$ and $B_{1}=20.714111$ respectively. The partner potential is calculated from Equation (23), using the numerical value of $B_{0}$. Since $V_{\text {eff }}^{(2)}(r)$ is no more analytically solvable, we solve the corresponding Schrödinger equation numerically by RungaKutta algorithm with appropriate boundary conditions for $r \rightarrow 0$ and $r \rightarrow \infty$. We checked that this potential supports only one bound state, whose binding energy is $B_{1}$, to within numerical error. Furthermore, the calculated eigenfunction agrees with the second of Equation (11), within numerical errors. We also solved Equation (2) by the Runga-Kutta algorithm and verified that the energies and wave functions of the ground and excited states were reproduced correctly within numerical errors. These indicate the accuracy of the numerical procedure. Finally we obtained $\psi_{E}^{(1)}(r)$ and $\psi_{E}^{(2)}(r)$ in the range $0<E<1$, by the Runga-Kutta algorithm, subject to $\psi_{E}^{(i)}(0)=0, \quad 0.3 \mathrm{~cm}(i=1,2)$. Phase shifts were calculated using Equation (25). Calculated phase shifts satisfy the supersymmetric relation Equation (20), within estimated numerical errors, when $\delta_{l}^{(1)}(k)$ and $\delta_{l+1}^{(2)}(k)$ are obtained from the solutions of the Schrödinger equation with $V_{\text {eff }}^{(1)}(r)$ and $V_{\text {eff }}^{(2)}(r)$ respectively. We have verified that the relationship Equation (20) is not valid between the phase shifts produced by $V_{\text {eff }}^{(1)}(r)$ and

$$
\left[V^{(2)}(r)+\frac{\hbar^{2}}{2 m} \frac{(l+1)(l+2)}{r^{2}}\right] \text {. }
$$

We again stress that for the $l=0$ effective potential $V_{\text {eff }}^{(1)}(r) \quad(=V(r))$, the partner potential $V_{\text {eff }}^{(2)}(r)$ is given by Equation (23), without any additional l-dependence. 
As a further check, we verified these relations by a numerical calculation for the Pöschl-Teller potential [4], which is an SIP. Thus the phase shift relation is valid whether the potential is an SIP or not, when $V_{\text {eff }}^{(2)}(r)$ is obtained as the supersymmetric partner of $V_{\text {eff }}^{(1)}(r)$, with no change in the l value, even though it may appear from Equation (20) that the phase shift for the partner potential should be for the $(l+1)$-th partial wave. The calculated results are presented in Tables $\mathbf{1}$ and $\mathbf{2}$ for the square well and Pöschl-Teller potentials, respectively.

\section{Conclusion}

In this work, we have critically examined the relationship between phase shifts produced by two supersymmetric (SUSY) partner potentials. A simple analytic reation, Equation (20), holds for both SIP and non-SIP, which relates the phase shift produced by the $l$-th partial wave of $V^{(1)}(r)$, i.e. by the potential

$$
V_{e f f}^{(1)}(r)=V^{(1)}(r)+\frac{\hbar^{2}}{2 m} \frac{l(l+1)}{r^{2}}
$$

and that by its SUSY partner $V_{e f f}^{(2)}(r)$. The latter phase shift corresponds to the $(l+1)$-th partial wave. However, there is no relation between phase shifts produced by

$$
V_{\text {eff }}^{(1)}(r) \text { and } V^{(2)}(r)+\frac{\hbar^{2}}{2 m} \frac{(l+1)(l+2)}{r^{2}},
$$

Table 1. Results of our calculation for the square well potential. $\delta_{l}^{(1)}(k)$ and $\delta_{l+1}^{(2)}(k)$ stand for the phase shifts produced by the potentials $V_{\text {eff }}^{(1)}(r)$ and $V_{\text {eff }}^{(2)}(r)$, respectively.

\begin{tabular}{ccccc}
\hline Energy $(E)$ & $\delta_{l}^{(1)}(k)$ & $\delta_{l+1}^{(2)}(k)$ & $\delta_{l}^{(1)}(k)-\delta_{l+1}^{(2)}(k)$ & $\tan ^{-1}\left(\frac{\gamma_{0}}{k}\right)$ \\
\hline 0.10000000 & -0.27066200 & 1.34833100 & 1.52259965 & 1.52231290 \\
0.20000000 & -0.38196900 & 1.25693000 & 1.50269365 & 1.50228403 \\
0.30000000 & -0.46682000 & 1.18731400 & 1.48745865 & 1.48695173 \\
0.40000000 & -0.53787800 & 1.12906600 & 1.47464865 & 1.47405634 \\
0.50000000 & -0.60005800 & 1.07814400 & 1.46339065 & 1.46272181 \\
0.60000000 & -0.65588400 & 1.03246800 & 1.45324065 & 1.45249847 \\
0.70000000 & -0.70685900 & 0.99080400 & 1.44392965 & 1.44311896 \\
0.80000000 & -0.75396300 & 0.95234500 & 1.43528465 & 1.43440894 \\
0.90000000 & -0.79787700 & 0.91652900 & 1.42718665 & 1.42624721 \\
1.00000000 & -0.83909800 & 0.88294800 & 1.41954665 & 1.41854544 \\
\hline
\end{tabular}

Table 2. Results of our calculation for the Pöschl-Teller potential. $\delta_{I}^{(1)}(k)$ and $\delta_{l+1}^{(2)}(k)$ stand for the phase shifts produced by the potentials $V_{\text {eff }}^{(1)}(r)$ and $V_{\text {eff }}^{(2)}(r)$, respectively.

\begin{tabular}{ccccc}
\hline Energy $(E)$ & $\delta_{l}^{(1)}(k)$ & $\delta_{l+1}^{(2)}(k)$ & $\delta_{l}^{(1)}(k)-\delta_{l+1}^{(2)}(k)$ & $\tan ^{-1}\left(\frac{\gamma_{0}}{k}\right)$ \\
\hline 0.10000000 & 1.16132000 & -0.10319000 & 1.26451000 & 1.26451896 \\
0.20000000 & 1.14997100 & -0.00025100 & 1.15022200 & 1.15026199 \\
0.30000000 & 0.79424100 & -0.27541900 & 1.06966000 & 1.06970331 \\
0.40000000 & -1.53982200 & 0.59497400 & 1.00679665 & 1.00685369 \\
0.50000000 & -1.28742700 & 0.89890900 & 0.95525665 & 0.95531662 \\
0.60000000 & 0.71413300 & -0.19753900 & 0.91167200 & 0.91173829 \\
0.70000000 & 0.84755500 & -0.02647400 & 0.87402900 & 0.87409797 \\
0.80000000 & -1.18922000 & 1.11268500 & 0.83968765 & 0.84106867 \\
0.90000000 & 0.68730200 & -0.12435500 & 0.81165700 & 0.81172612 \\
1.00000000 & 0.04691800 & -0.73736500 & 0.78428300 & 0.78539816 \\
\hline
\end{tabular}


where $V^{(2)}(r)$ is the SUSY partner of $V^{(1)}(r)$. It would be wrong to take $\delta_{l+1}^{(2)}(k)$ to be the phase shift produced by the $(l+1)$-th partial wave in $V^{(2)}(r)$. Our numerical calculation of the phase shifts produced by a non-SIP as well as an SIP confirm this. It would be of great interest, if the phase shift could be obtained algebraically. This would be possible, if $V_{\text {eff }}^{(1)}(r)$ were shape invariant. But, even if $V^{(1)}(r)$ is shape invariant, i.e. $V^{(1)}(r)$ and $V^{(2)}(r)$ have same mathematical shapes, in general $V_{\text {eff }}^{(1)}(r)$ is not shape invariant. Thus, in general the phase shift cannot be obtained algebraically, even for standard SIPs. Since Equation (20) relates $\delta_{l+1}^{(2)}(k)$ with $\delta_{l}^{(1)}(k)$, an algebraic derivation of the phase shift is possible if $\delta_{l+1}^{(2)}(k)$ and $\delta_{l}^{(1)}(k)$ relate to potentials which have the same mathematical shape. This is possible, if SUSY partner of $V_{\text {eff }}^{(1)}(r)$ explicitly corresponds to the $(l+1)$ partial wave, i.e. $l$ is the shape invariance parameter of Equation (1) and $f(l)=l+1$. Such a restrictive condition is valid only for the Coulomb potential [4], only for which analytic expression for the phase shift is possible [5-7].

\section{Acknowledgements}

The author wishes to thank Prof. T. K. Das for his help- ful discussions and suggestions.

\section{REFERENCES}

[1] E. Witten, "Dynamical Breaking of Supersymmetry," Nuclear Physics B, Vol. 188, No. 3, 1981, pp. 513-554. doi:10.1016/0550-3213(81)90006-7

[2] F. Cooper and B. Freedman, "Aspects of Supersymmetric Quantum Mechanics," Annals of Physics, Vol. 146, No. 2, 1983, pp. 262-288. doi:10.1016/0003-4916(83)90034-9

[3] Y. A. Gel'fand and E. P. Likhtman, "Extension of the Algebra of Poincare Group Generators and Violation of P Invariance," JETP Letters, Vol. 13, 1971, p. 323.

[4] F. Cooper, A. Khare and U. Sukhatme, "Supersymmetry and Quantum Mechanics," Physics Reports, Vol. 251, No. 5-6, 1995, pp. 267-385. doi:10.1016/0370-1573(94)00080-M

[5] C. V. Sukumar, "Supersymmetric Quantum Mechanics and the Inverse Scattering Method," Journal of Physics A, Vol. 18, No. 15, 1985, p. 2937. doi:10.1088/0305-4470/18/15/021

[6] J. J. Sakurai, "Modern Quantum Mechanics," AddisonWesley, Boston, 2000.

[7] S. Mahapatra and T. K. Das, "On the Algebraic Derivation of Phase Shifts for Shape Invariant Potentials," Modern Physics Letters A, Vol. 26, No. 23, 2011, pp. 1753-1761. doi:10.1142/S0217732311036061 